\title{
Ecosistema del emprendimiento en la Región Caribe Colombiana
}

\author{
Entrepreneurship Ecosystem in the Colombian Caribbean Region
}

\author{
Diego Cardona-Arbeláez* \\ Colegio Mayor de Bolívar - Colombia \\ ORCID iD: https://orcid.org/0000-0002-9123-0156 \\ dcardona@colmayorbolivar.edu.co

\section{Gioryanis Tapias-Pacheco} \\ Universidad Tecnológica de Bolívar - Colombia \\ ORCID iD: https://orcid.org/0000-0002-6346-6970 \\ gtapias@utb.edu.co
}

* Autor a quien debe ser dirigida la correspondencia
Fecha de recepción: 03/02/2021

Fecha de evaluación: 19/03/2021

Fecha de aceptación: 02/05/2021
Cómo citar: Cardona-Arbeláez, D., \& Tapias-Pa-
checo, G. (2021). Ecosistema del emprendimiento en
la región caribe colombiana. Revista Cientifica Anfi-
bios, $4(1) . \quad$ https://doi.org/10.37979/afb.2021v4n1.82

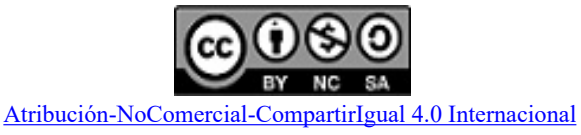

\section{Resumen}

En gran medida se ha hablado de la importancia del desarrollo de emprendimientos para la dinámica económica y social de un país o una región en particular; es de suma importancia entender el porqué de la necesidad de crear emprendimientos que generen cambios significativos en la realidad de la sociedad en la cual se ejecutan y con esto buscar las estrategias que permitan su sostenimiento. Se realiza una revisión de la literatura referente al desarrollo de ecosistemas de emprendimiento en diferentes territorios con el fin de presentar una reflexión en cuanto a las necesidades que existen en la ciudad de Cartagena para la adecuada articulación y fomento de emprendimientos de alto impacto. A manera de reflexión se concluye que Cartagena cuenta con los pilares necesarios para conformar un ecosistema de que fomente el emprendimiento de alto impacto pero es necesario que se trabaje desde las instituciones en la erradicación de la pobreza y se fomente la cultura emprendedora.

\section{Palabras Clave}

Ecosistema de emprendimiento; emprendimiento de alto impacto; startup; región caribe colombiana; desarrollo

\begin{abstract}
Largely it has spoken of the importance of development of entrepreneurship for economic and social dynamics of a country or a particular region; It is very important to understand why the need to create ventures that generate significant changes in the reality of the society in which you run and look for strategies to their support. A review of the literature on the development of entrepreneurial ecosystems in different territories is carried out in order to present a reflection on the needs that exist in the city of Cartagena for the adequate articulation and development of high impact ventures. As a reflection, it is concluded that Cartagena has the necessary pillars to form an ecosystem that encourages high-impact entrepreneurship, but it is necessary to work from the institutions in the eradication of poverty and foster an entrepreneurial culture.
\end{abstract}




\section{Keywords:}

Entrepreneurship ecosystem; high-impact entrepreneurship; startup; colombian caribbean region; development

\section{Introducción}

Actualmente está siendo foco de estudio los emprendimientos dinámicos o de alto impacto, los cuales han sido llamados también como empresas de crecimiento rápido o gacelas (Almus, 2002), por emprendimientos dinámicos se entiende como aquellos proyectos y nuevas empresas que tienen el potencial y las perspectivas de convertirse, luego de los primeros años de vida, en nuevas pymes competitivas y seguir creciendo. (Kantis, Federico J, \& Garcia S.I, 2016). De este modo todo depende del enfoque de su definición y desde qué perspectiva se mire, por lo tanto el emprendimiento tiene infinidades de definiciones. De acuerdo con (Zambrano-Vargas \& Vázquez-García, 2019) el emprendimiento es un concepto emergente dentro de la administración, que ha tomado gran importancia por ser agente de cambio social. El Ministerio de Comercio, Industria y Turismo de Colombia define el emprendimiento como el conjunto de personas, variables y factores que intervienen en el proceso de crear una empresa, su resultado es la creación de valor que beneficia a la empresa, la economía y la sociedad. (MINCIT, s.f.).

Los emprendimientos de alto impacto son de suma importancia para la diversificación del tejido productivo de una región, introducir innovaciones y generar empleo de calidad (Kantis, Federico, \& Menendez, 2012). Para el adecuado desarrollo de emprendimientos de alto impacto es necesario contar con un ecosistema que propicie los mismos, de este modo, un ecosistema de emprendimiento es una metáfora que hace referencia a ese conjunto de actores entre los que se encuentran instituciones formales, en las cuales sus líderes o miembros tienen la intención explícita de fomentar el emprendimiento (Agudelo, 2016).

El primer acercamiento a la metáfora de ecosistemas de emprendimientos fue hecha por James Moore (1993) en un artículo publicado en la Harvard Business Review en 1993, titulado "Predators and Prey: A New Ecology of Competition", donde señala que un ecosistema de negocios es aquel espacio donde hay una interconexión y dependencia mutua entre los agentes económicos, y la prosperidad colectiva era fundamental para el éxito y la supervivencia de las compañías.
Así mismo, un ecosistema sano es el espacio en donde se produce la integración entre los diferentes jugadores que lo articulan, generando oportunidades para el desarrollo económico de una ciudad, una región y un país. (Reinoso Lastra \& Uribe, Emprendimiento y Empresarismo, 2013).

Una mayor tasa de emprendimiento impulsa el crecimiento económico, que se evidencia en un crecimiento del PIB; además, disminuye la tasa de desempleo, cuando estas nuevas empresas se convierten en una forma de autoemplearse y, cuando empiezan a crecer y a generar otros empleos (Audretsch y Thurik, 2001. Citado en López, 2016).

Desde esta perspectiva, se presenta la oportunidad futura de idear y diseñar respuestas estratégicas que permitan solucionar los problemas estructurales y de enfoque que presentan actualmente la actividad en el país; y por lo mismo, incorporar en el ejercicio de funciones y en las corrientes de opinión de los distintos estamentos de las organizaciones una visión, desde lo conceptual, teórico y académico, diferente a la que se tiene hoy de la forma cómo se están direccionando estos ecosistemas emprendedores de gran importancia para la economía y desarrollo de local y regional.

Por otro lado, la identificación de los factores clave para articular sinérgicamente el emprendimiento de alto impacto en el ecosistema empresarial de la ciudad de Cartagena, permitirá aprender de las experiencias de los ecosistemas más exitosos a nivel mundial, replicando las experiencias de éxito. Lo cual permitirá formular estrategias que faciliten establecer el derrotero hacia el éxito de las empresas (Villanueva Imitola, 2017) y que permitan potenciar las fortalezas que actualmente posea el tejido empresarial.

En Colombia, las redes regionales de emprendimiento son creadas con el fin de fomentar el emprendimiento, las mismas son presididas por las gobernaciones y la secretaría técnica la ejerce las cámaras de comercio de la región (MinCIT, 2017); el deber de estas redes regionales de emprendimiento es el promover la generación de emprendimientos, pero se evidencia de manera 
empírica que la ciudad de Cartagena actualmente no cuenta con una red estructurada y que existe una alta volubilidad en el sostenimiento de las acciones que se ejecutan para su desarrollo. Se necesita la articulación de las empresas, las instituciones, la sociedad y las instituciones gubernamentales.

En este artículo se presenta una reflexión hacia lo que debe ser un ecosistema que fomente el emprendimiento de alto impacto en la ciudad de Cartagena, teniendo en cuenta que es la cuarta ciudad industrial del país, dónde confluyen un gran desarrollo logistico, portuario, y es el primer destino (Cartagena en cifras, 2017), con sectores altamente dinámicos como industria, turismo, puertos y construcción; pero que en materia de emprendimiento se encuentra rezagada.

\section{Antecedentes del tema}

El emprendimiento en las regiones es tomado como referente de desarrollo económico y social, de allí la importancia de su promoción, fomento y protección; su gestión procura el desarrollo nacional, donde cada región aporta de manera significativa al crecimiento, proyectando a la nación a una economía global de alta competitividad (Romero, 2017).

Los emprendimientos de alto impacto son de suma importancia para la diversificación del tejido productivo de una región, introducir innovaciones y generar empleo de calidad (Kantis, Federico, \& Menendez, 2012). El impacto del emprendimiento y la innovación sobre los negocios avanza de manera rápida, la evidencia internacional confirma que los niveles más altos de desarrollo corresponden a países que muestran también altos niveles de emprendimiento innovador en sus economías.

Birch (1987) afirma que las empresas han de considerarse dinámicas cuando se cumple que sus ventas presentan un crecimiento del 15\% - 20\% o más por año, esto se debe cumplir para varios periodos seguidos. Entonces una empresa se denominará gacela (o de alto impacto) cuando el crecimiento ocurre en sus primeros años de vida ( 3 a 5 años iniciales), también se conocen como "nuevas empresas dinámicas" y llegan a convertirse en pymes.

Cartagena es una de las ciudades con mayor aporte al producto interno bruto (PIB) del país con sectores altamente dinámicos, el aporte del departamento de Bolívar es del 4,2\% al 2017, donde su capital tiene una participación del 68,3\% gracias a su condición turística, portuaria e industrial (Informe económico de los municipios de la jurisdiccion de la cámara de comercio de Cartagena, 2020).

En contraste, es una ciudad que presenta grandes brechas en cuanto a su desarrollo social, debido a sus altos niveles de pobreza para 2016 los indicadores de pobreza monetaria y pobreza monetaria extrema aumentaron respecto al promedio nacional, $29,1 \%$ y 5,5\% respectivamente, ubicando a Cartagena como la tercera ciudad del país con más pobreza (Ayala García \& Meisel Roca, 2016).

Cartagena de Indias como una de las ciudades principales de Colombia, que se destaca por su turismo e industria (Banco de la Republica, 2016), debe contar con un ecosistema consolidado que permita fomento, promoción y protección de los emprendimientos de alto impacto, el cual actualmente no se percibe ni está en funcionamiento; la red de emprendimiento de Bolívar que debería ser la figura que opera el proceso de articulación para su desarrollo no está activa.

Teniendo en cuenta lo antes dicho, en Cartagena según Orozco y Arraut (2013), las políticas gubernamentales para la creación de empresas se consideraban deficientes, es decir, no favorecían claramente a las empresas de nueva creación y tampoco son una prioridad para el Gobierno. En el caso de la legislación tributaria se considera como barrera para crear empresas e impulsar el crecimiento de las empresas. En ese mismo sentido la estructura tributaria, es decir, a las tasas e impuestos y otras regulaciones, no está aplicada de forma coherente y predecible, lo cual desfavorece la creación de nuevas empresas. En cuanto a lo privado, los mismos autores (Orozco y Arraut, 2013), dicen que el financiamiento para nuevas empresas en la ciudad es muy deficiente.

Por el lado de la sociedad, y en cuanto a la cultura empresarial e innovadora, Orozco y Arraut (2013), señalan que en la ciudad se da reconocimiento del emprendedor como un actor importante en la sociedad, la percepción acerca de las oportunidades, la apertura del consumidor a probar nuevos productos o servicios dados por la percepción de oportunidades, la cobertura de los medios de comunicación, entre otras variables. 
En este sentido, en el último estudio realizado por la GEM (Global Entrepreneurship Monitor Caribe) en el 2012, muestra que el $81.7 \%$ de la población percibe que existen buenas oportunidades para iniciar un negocio en la ciudad, en tanto que $61.3 \%$ considera que posee los conocimientos y las habilidades necesarios para hacerlo.

En este análisis la academia es la que sale mejor librada, según Orozco y Arraut (2013), unas de las fortalezas que permiten la competitividad del emprendimiento en la ciudad, son la educación superior y los sistemas de formación profesional, esto es reflejo de las iniciativas de las universidades para fomentar en sus estudiantes el emprendimiento a través de varias estrategias, incluidas las cátedras empresariales y las opciones de graduación con propuestas empresariales.

Sumado a lo anterior, el indicador Doing business (2017), que mide en clima de negocios; es decir, cuán rápido es crear una empresa en Cartagena, también da una idea de cómo funciona el ecosistema emprendedor de la ciudad. Este indicador mide cuatro aspectos: tiempo y número de trámites, pago de impuestos, y la obtención de permisos de construcción.

Para el caso de Cartagena en tiempo y número de trámites para apertura de empresas se mantienen y la facilitación de negocios se pasó del 10 al 11 puesto, en pago de impuestos del 22 al 26; en los costos de apertura de empresas, y la facilitación para la obtención de permisos de construcción donde se pasó del 16 al 22; para tener finalmente un resultado global de 17 pero en tres de los cuatro índices la ciudad ocupo puestos por encima del 20 (Doing business, 2017). Se puede concluir, que el ecosistema emprendedor de Cartagena debe mejorar su desempeño global.

De otro lado, está el emprendimiento dinámico, según Lozano (2013), emprendimiento de alto Impacto se define como un emprendimiento que es rentable, crece rápida y sostenidamente, y que es capaz de lograr un nivel de ventas bastante significativo en una década. En este mismo sentido Kantis, Federico, y Menéndez (2012), señalan como emprendimiento de alto Impacto las nuevas empresas cuyo crecimiento les permite abandonar en pocos años el mundo de la microempresa para transformarse en Pymes competitivas con potencial y proyección de seguir creciendo.

\section{Emprendimiento en América del norte}

En la parte norte del continente Americano se encuentran 9 de los ecosistemas más potentes del mundo (Startupgenome, 2017), compuesto por los ecosistemas de Silicon Valley, New York, Boston, Los Ángeles, Seattle, Austin y Chicago, en Estados Unidos y los de Vancouver y Toronto en Canada, la mentalidad, la alta competencia, un fuerte networking y una legislación particular son características que el ecosistema emprendedor de Estados Unidos tiene (Foundersandtips). Estados Unidos lidera el mundo en el emprendimiento, y es el primero en la región de América del Norte, justo por delante de Canadá (Zoltán J. Ács, László Szerb, Erkko Autio, 2015).

El modelo cultural de emprendimiento en Norteamérica da prioridad a los valores del dominio y la autonomía (Marta Peris-Ortiz, 2017), concepto basado en la teoría de Shalom H. Schwartz, (Torres, 2016) donde señala que la cultura norteamericana enfatiza el dominio y la autonomía afectiva y pone poco el acento en la armonía, esto quiere decir que esta cultura da prioridad a perseguir sus intereses individuales y de acuerdo al Global Entrepreneurship index los factores más importante que componen el valor para Estados Unidos son la capacitación inicial, el capital humano, alto crecimiento y la internacionalización, en cuanto a las instituciones de propiedad privada y la ejecución de contratos dan a los empresarios el incentivo para invertir en capital físico y humano, combinar insumos para crear nuevas funciones de producción y para completar los mercados.

\section{Emprendimiento en América Latina}

Indicadores como los del Reporte Global de Competitividad del Foro Económico Mundial o estudios como el Doing Business, reflejan las grandes diferencias respecto a las condiciones para realizar negocios entre los países de Latinoamérica (Hidalgo, Kamiya, \& Reyes, 2014).

Estas diferencias se ven expresadas, en los costos y plazos para la apertura de un negocio en cada uno de los países. En concreto, Chile y Brasil son los países donde el costo económico de abrir un negocio es menor, ya que este representa entre un 4,5\% y un 4,8\% del PIB per cápita (Hidalgo, Kamiya, \& Reyes, 2014). La realidad es muy distinta en otros países, siendo el caso ex- 
tremo el de Honduras, donde el costo equivale al $100,6 \%$ del ingreso per cápita, lo que ciertamente disuade a muchos de participar de la economía formal. (Hidalgo, Kamiya, \& Reyes, 2014).

Las mediciones en la actividad emprendedora temprana (TEA) de Latinoamérica oscilan entre el $36 \%$ de proporción que muestra Ecuador y el $14 \%$ de México con mediciones intermedias como el $24,3 \%$ de Chile y el $17,3 \%$ de Brasil. Por otra parte, el mismo estudio da cuenta de la proporción de la población adulta que está iniciando un emprendimiento y que tiene altas expectativas de crecimiento, esto es, que espera generar $20 \mathrm{o}$ más empleos en los próximos 5 años. La proporción más elevada en la medición está en Colombia, donde el 58,3\% de los emprendedores tiene o ha iniciado un negocio, seguido de Chile, con el $36 \%$ y Trinidad y Tobago con $32 \%$. Por el contrario, los emprendedores con altas expectativas de crecimiento son muy pocos, Guatemala (4\%) y Brasil (10\%), mientras países como Perú se encuentran en posiciones relativamente bajas, al alcanzar 20\%. (Hidalgo, Kamiya, \& Reyes, 2014).

\section{Emprendimiento en Colombia}

En la región latinoamericana, Colombia se encuentra clasificado como el tercer país con mejor economía que facilita hacer negocios según el ranking Doing Business, detrás de Puerto Rico (Banco Mundial, 2019).

En cuanto a las políticas públicas, Colombia cuenta con la Ley 1014 de 2006 de fomento a la cultura del emprendimiento, se fundó la red nacional para el emprendimiento, responsable de formular la política pública de emprendimiento, presidida por el Ministro de Comercio, Industria y Turismo, y con participación activa de otros ministerios relacionados con la materia, las Instituciones de Educación Superior, el Servicio Nacional de Aprendizaje, Bancoldex, organizaciones del sector privado, financiero y representantes de los emprendedores. Dicha red ha formulado la Política Nacional de Emprendimiento y sus ajustes.

Así mismo, en Colombia se crearon Redes Regionales para fomentar el emprendimiento en los departamentos presididas por las Gobernaciones y cuya secretaria técnica es ejercida por las Cámaras de Comercio. Este trabajo de articu- lación institucional se destaca entre los países de América Latina, aunque tiene espacios de mejora y fortalecimiento para el diseño de nuevos instrumentos y programas de emprendimiento acorde con las diferencias de las regiones colombianas (Hidalgo, Kamiya, \& Reyes, 2014).

\section{Emprendimiento en Cartagena de Indias, distrito turístico, histórico y cultural}

A 2018, Cartagena cuenta con 1.028.736 habitantes según las proyecciones de población del DANE, lo que la clasifica como la quinta ciudad más grande del país después de Barranquilla que tiene un total de habitantes de 1.223.616, Cali con 2.394.925, Medellín 2.486.723 y Bogotá 7.980.001 (Gran Encuesta Integrada de Hogares, 2018).

De acuerdo a la cámara de comercio de Cartagena, durante el 2019 se inscribieron 8.651 empresas nuevas, un 1,3\% menos que lo registrado en el año 2018. No obstante, el crecimiento promedio en los últimos 3 años fue de $5 \%$, y los sectores económicos con mayor creación de empresas fueron comercio $(33,1 \%)$, alojamiento y servicios de comida $(14,4 \%)$, industrias manufactureras $(8,5 \%)$ y actividades artísticas, de entretenimiento y recreación (8,3\%) (Cartagena en cifras, 2019).

El Global Entrepreneurship Monitor (GEM, 2012) estudia la actividad empresarial de Cartagena. En ese informe se evidencia el estado de la actividad empresarial y se muestran las principales características, actitudes y aspiraciones de los individuos involucrados en el proceso empresarial, entre los cuales se destacan las siguientes conclusiones:

La actividad empresarial de Cartagena, medida con la tasa de nueva actividad empresarial (TEA, por sus siglas en inglés), muestra que el $17.2 \%$ de la población adulta está involucrada en la creación de nuevas empresas. Además, solo el $5.9 \%$ logró superar los 42 primeros meses en el mercado para llegar a la etapa de empresarios establecidos, cifra inferior al promedio nacional. También se resalta como resultado desfavorable para la ciudad que un porcentaje significativo $(4.0 \%)$ de empresarios se retiró del proceso, principalmente por problemas financieros. 
Se destaca como hecho positivo que la oportunidad se ha establecido como el principal motivo para emprender en Cartagena, desde que se empezó a realizar el estudio GEM tanto en esta ciudad como en el país. Es así como el $14.3 \%$ de la actividad empresarial está basada en emprendimientos por oportunidad y solo el $2.9 \%$ por necesidad.

Con relación a la caracterización de los cartageneros involucrados en estas actividades, se evidencia que en 2012 la mayor parte de las nuevas empresas de la ciudad son creadas mayoritariamente por mujeres $(17.3 \%)$, en tanto que solo el $16.1 \%$ de los hombres lo hace. Sin embargo, la tasa de hombres cuyas empresas llevan más de 42 meses en funcionamiento $(3.3 \%)$ es superior a la de mujeres $(2.6 \%)$.

En lo referente a las actitudes de los individuos frente a la creación de empresas, los resultados son favorables para Cartagena en 2012. En efecto, $81.7 \%$ de la población percibe que existen buenas oportunidades para iniciar un negocio en la ciudad, en tanto que $61.3 \%$ considera que posee los conocimientos y las habilidades necesarios para hacerlo.

En cuanto al desarrollo del ecosistema de emprendimiento, la ciudad de Cartagena ha ido desarrollando desde el 2000, alianzas públicas privadas que han permitido a la fecha de hoy consolidar una apuesta empresarial, que permita generar nuevas unidades productivas. El primer avance fue la creación de Incubar Bolívar (en la actualidad ya no existe).

\section{Referentes para análisis del fomento em- prendimiento}

\section{Definición de emprendimiento}

La palabra emprender proviene del francés entrepernar que significa iniciar algo (Ramírez Rodríguez, 2009), a partir del siglo XVII y XVIII se nombraba como emprendedor toda aquella persona que iniciaba un gran proyec- to. La misma fue introducida por vez primera al diccionario de la real academia de la lengua española en 1732, definiendo entonces como la persona que se determina a hacer algo.

Para Shumpeter (1950) el emprendimiento era representado por una persona con características extraordinarias que promovía las ideas innovadoras, reconociendo al emprendedor como el eje de una economía, de acuerdo a sus apreciaciones la función de los emprendedores es reformar o revolucionar el patrón de producción generando nuevos productos o servicios a través de la creatividad, o más comúnmente, una posibilidad técnica no probada, para producir un nuevo producto o uno viejo de nueva manera; o proveer de una fuente de insumos o un material nuevo; o reorganizar una industria, entre otras.

Por otra parte Vesga (2009) define el emprendimiento como una actitud básica hacia la identificación de oportunidades y la toma de riesgos por parte de los individuos, en organizaciones de todos los tamaños.

\section{Emprendimiento de Alto Impacto}

Los emprendimientos de alto impacto son de suma importancia para la diversificación del tejido productivo de una región, introducir innovaciones y generar empleo de calidad (Kantis, Federico, \& Menendez, 2012). El impacto del emprendimiento y la innovación sobre los negocios avanza de manera rápida, la evidencia internacional confirma que los niveles más altos de desarrollo corresponden a países que muestran también altos niveles de emprendimiento innovador en sus economías. (Vesga, 2009)

La operación de la economía del emprendimiento y la innovación se puede entender mejor al considerar tres niveles diferentes y las interacciones entre ellos: el nivel macro, el nivel de las organizaciones y el nivel de los individuos. Estas interacciones se pueden apreciar en la siguiente figura: 


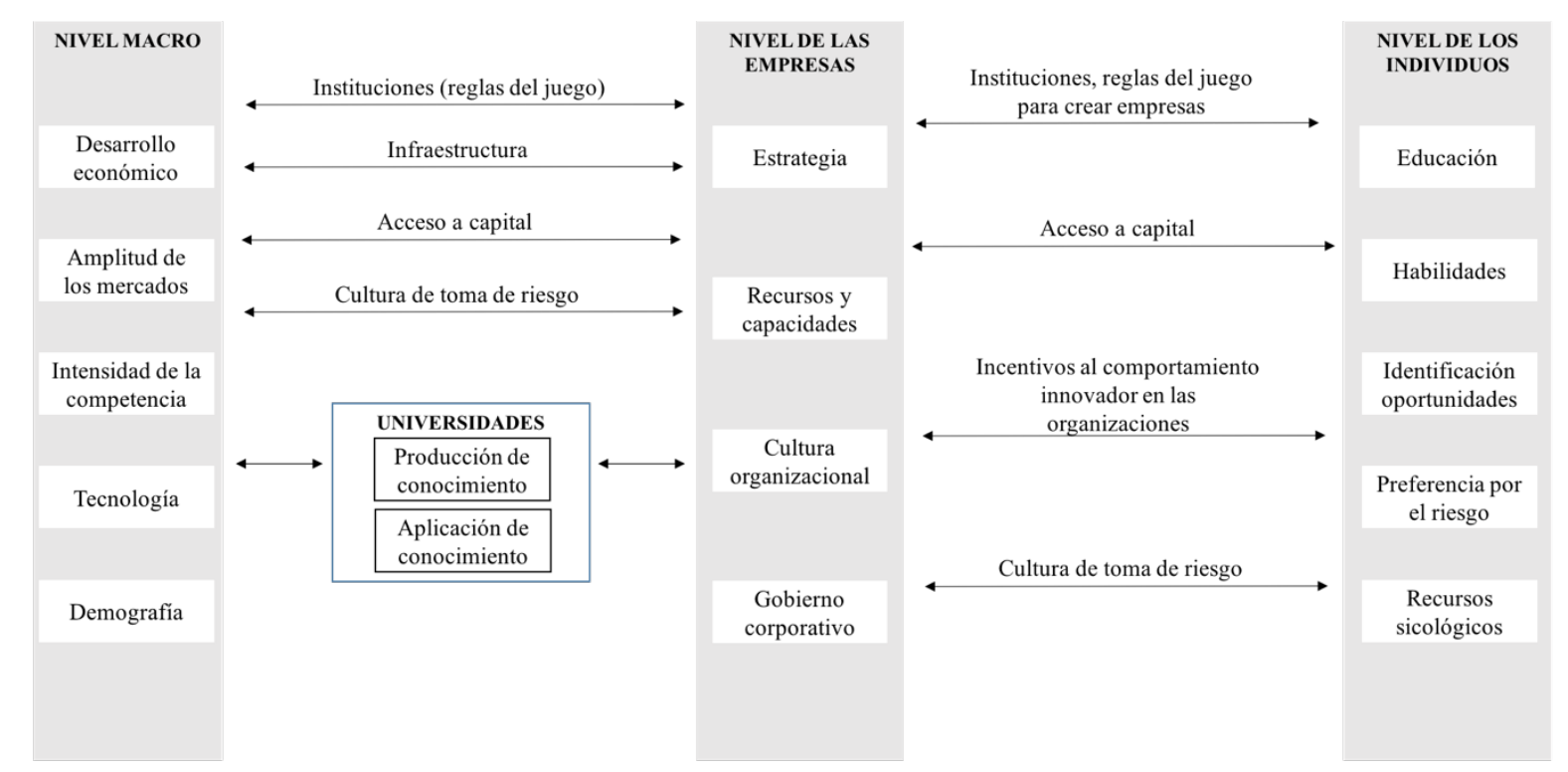

Figura 1. Emprendimiento y la Innovación en un país. Tres niveles de análisis Fuente: Adaptado de Rafael Vesga, emprendimiento e innovación en Colombia, pág. 4.

\section{Actividad Emprendedora}

Se considera que la actividad emprendedora es un conjunto de iniciativas empresariales que fomentan el cambio innovador. En esta línea se pronuncia Audretsch David Bruce (1995) para quien el estímulo emprendedor consiste en "un proceso de cambio". También la propia OCDE (2003) define el estímulo emprendedor en este sentido considerando que los empresarios son agentes del cambio y de crecimiento de una economía del mercado, siendo quienes pueden acelerar la generación y del crecimiento de una economía de mercado, siendo quienes pueden acelerar la generación, difusión, y el uso de la idea innovadora.

El GEM (Global Entrepreneurship Monitor) establece cuatro etapas en el llamado "proceso de creación de empresas" a partir de la cual, analizan los resultados en términos del potencial de los individuos para emprender, de la Tasa de Actividad Emprendedora (emprendedores nacientes y nuevos empresarios) y de la Tasa de Empresarios Establecidos. En consecuencia percibe tres momentos en el proceso: Concepción, Nacimiento y Persistencia.

\section{Ecosistema de emprendimiento}

Un ecosistema de emprendimiento es una metáfora que hace referencia a ese conjunto de actores entre los que se encuentran instituciones formales, en las cuales sus líderes o miembros tienen la intención explicita de fomentar el emprendimiento (Agudelo, 2016). El primer acercamiento a la metáfora de ecosistemas de emprendimientos fue hecho por James Moore (1993) en un artículo publicado en la Harvard Business Review en 1993, titulado "Predators and Prey: A New Ecology of Competition", donde señala que un ecosistema de negocios es aquel espacio donde hay una interconexión y dependencia mutua entre los agentes económicos, y la prosperidad colectiva era fundamental para el éxito y la supervivencia de las compañías.

Un ecosistema sano en donde se produzca la integración entre los diferentes jugadores que lo articulan genera oportunidades para el desarrollo económico de una ciudad una región y un país, cuando se habla de emprendimiento se parte de la persona para llegar a su contexto, este es personal y social, se inicia con la identificación y potencialización personal, para orientarlo hacia dimensiones sociales por sus efectos (Reinoso Lastra \& Uribe, Emprendimiento y Empresarismo, 2013). 
El Global Entrepreneurship index (2017) presenta una visión adicional respecto a la caracterización de un ecosistema emprendedor, en donde explica que un sistema es un conjunto organizado de subsistemas interactivos e interdependientes que funcionan juntos como un todo para lograr un propósito.

Según Daniel Isenberg (2010) de Babson College un ecosistema de emprendimiento además de redes de personas y organizaciones se compone de varios dominios: una cultura favorable a la innovación, liderazgos claros, capital humano de calidad, mercados desarrollados y una gama de apoyos institucionales y de infraestructura tanto públicos como privados

\section{Teorías de Emprendimiento}

\section{Teoría del crecimiento de Schumpeter (1942)}

Schumpeter (1942) alude que el crecimiento económico propiamente dicho no es suficiente sino que se debe tender al desarrollo económico, lo que conlleva a un incremento de gran impacto en el bienestar social y económico, esto a través de la "destrucción creadora" impulsada fundamentalmente por nuevos emprendedores.

En tal sentido, Schumpeter (1949) analiza la actividad empresarial como la innovación y no la imitación y concluye que el emprendedor mueve la economía fuera del equilibrio estático. Schumpeter es el primero en formular el concepto de espíritu emprendedor, el mismo como un componente vital para el desarrollo económico de los países, y estado que afecta los ciclos financieros de una nación al presentar estados de evolución en las industrias o empresas.

\section{Teoría de crecimiento de las empresas de Gibrat (1931)}

La ley del efecto proporcional de Gibrat implica que el crecimiento es completamente aleatorio. Gibrat (1931) establece que las tasas de crecimiento de las empresas son independientes del tamaño. En términos estadísticos que la probabilidad de un cambio proporcional en tamaño durante un periodo especifico de tiempo es la misma para todas las empresas de un sector dado, independiente de su tamaño en el inicio del periodo especificado.

\section{Teoría de las gacelas (1987)}

David Birch (1987) fue el primero en referirse a la metáfora de empresas gacelas, entendiendo las mismas como aquellas que crecen más rápido que el promedio general; es importante comprender cuáles son los determinantes que hacen que crezcan más que el promedio por lo cual al mismo tiempo se convierte en una alta brecha de investigación (Zoltan Acs, 2006).

Birch (1987) afirma que las empresas han de considerarse dinámicas cuando se cumple que sus ventas presentan un crecimiento del $15 \%$ al $20 \%$ o más por año, entonces una empresa se denominará gacela cuando el crecimiento ocurre en sus primeros años de vida (3 a 5 años iniciales), también se conocen como "nuevas empresas dinámicas" y llegan a convertirse en pymes.

\section{Teorías institucionales y el desarrollo del emprendimiento (1990)}

La teoría institucional de North D. (1990), y Baumol (1993) plantea que la actividad em-prendedora está ligada a los factores del entorno en cual opera. Las instituciones son esas reglas sociales y restricciones que configuran la interacción humana. En consecuencia es la estructura que incentiva el intercambio humano, ya sea político social o económico. (North, 1990, p 3).

Según North (1990), los factores que influencian dinámicamente el proceso emprendedor pueden ser formales e informales. Por un lado, las políticas gubernamentales como por ejemplo: las leyes, constituciones o procedimientos para crear una empresa o las políticas son consideradas como factores formales del entorno. Mientras que los factores informales están definidos como los códigos de conducta, normas de comportamiento y actitudes, por ejemplo, el espíritu emprendedor, la cultura empresarial y las actitudes hacia el emprendimiento (North D. C., 1990).

Amartya Sen (1999) en su trabajo "el desarrollo como libertad" plantea la relación entre desarrollo social y la libertad individual, de este modo lo que una persona puede lograr es resultado de la influencia de las oportunidades económicas, libertades políticas, poderes sociales, condiciones adecuadas para buena salud y educación básica y el fomento y desarrollo de iniciativas. Así, el 
emprendimiento es visto como una actividad que desarrolla y genera libertad a las personas, además es visto como un bien social en cuanto debe ser promovido, fomentado y protegido por las políticas que den garantía del libre acceso a todos los habitantes.

Entre los autores analizados hasta aquí existe un elemento común, todos incluyen diferentes variables en el proceso, pero casi todos incluyen proximidad al sistema productivo, las redes empresariales, la vinculación Universidad-Empresa, y las redes laborales. Por tal motivo, resulta fundamental abordar el concepto de ecosistema emprendedor; este es el conjunto de agentes (entidades públicas, instituciones educativas y de investigación, empresas, inversores, sistema financiero, fundaciones, etc.), que operan en un determinado entorno geográfico, y que tienen un interés, real o potencial, en que haya más espíritu empresarial. Este ecosistema, favorece o inhibe el impulso de una persona para convertirse en un empresario, así como determinan las probabilidades de éxito después del lanzamiento de una nueva actividad (Alba, 2015)

\section{Evaluación anual del nivel nacional de acti- vidad emprendedora y empresarial}

El GEM (Global Entrepreneurship Monitor) es una red de investigación sin ánimo de lucro fundada en el año 1999 por el London Business School y el Babson College, para monitorear la actividad de creación de empresas en los países que hagan parte de ella.

El programa cubre tanto a países en desarrollo como desarrollados. El GEM, basado en la evaluación del nivel nacional de actividad emprendedora para todos los países partícipes, involucra la exploración del papel del emprendimiento en el crecimiento económico nacional.

Banco Mundial Proyecto Doing Business: mide lo fácil o difícil que es comenzar un negocio en una economía, por medio del registro de todos los procedimientos oficialmente requeridos o comúnmente hechos en la práctica por un emprendedor, para comenzar y operar formalmente un negocio industrial o comercial, así mismo, el tiempo y el costo requerido para completar estos procedimientos.
El informe Doing Business (2017) registra todos los procedimientos que se requieren oficialmente para que un empresario pueda abrir y operar formalmente una empresa industrial o comercial. Éstos incluyen la obtención de todas las licencias y permisos necesarios que se le exijan respecto de la empresa y sus empleados, y la realización de cualquier notificación, comprobación o inscripción ante las autoridades correspondientes. La clasificación en facilidad de apertura de una empresa, es el promedio simple de las clasificaciones percentiles de los indicadores que la componen.

En Colombia, desde la década de los setenta se viene hablando el tema del emprendimiento, pero solo hasta finales de la década de los noventa e inicios del 2000 se dan los primeros planes, leyes y políticas formuladas desde el gobierno nacional para promover la actividad emprendedora (Vásquez, 2013).

\section{Conclusiones}

A manera de reflexión se concluye que la ciudad de Cartagena cuenta con elemento esenciales para la construcción de un ecosistema que fomente de forma efectiva el emprendimiento de alto impacto; esto de acuerdo a las afirmaciones teóricas y la revisión del estado del arte, estudios que demuestran la capacidad de la ciudad en la construcción de redes y aplicación de políticas que lleven a los cartageneros a la construcción de una mentalidad activa en cuanto al desarrollo empresarial.

Es igualmente importante señalar que a pesar de que se cuenta con estos pilares sino se erradica la pobreza y la desigualdad social difícilmente proyectos de desarrollo al emprendimiento podrán ser consolidados, de acuerdo con Amartya Sen el desarrollo social conduce a la libertad y esta libertad puede ser expresada en la construcción de emprendimientos que deben ser fomentados en la construcción de una cultura alrededor de la misma.

Además de lo anterior, el desarrollo y fomento de espacios de reflexión hacia la generación de emprendimientos de alto impacto da las pautas necesarias para volver el tema algo fundamental en las agendas de los diferentes actores que componen el ecosistema de emprendimiento en la ciudad, lo que permitiría articularlos y lo que a su vez generaría la dinámica necesaria que se expone en 
el presente documento para convertir a Cartagena en el centro de emprendimiento dinámico del país.

De esta manera se puede considerar a Cartagena de Indias como una ciudad con altas posibilidades de generación de nuevas empresas, donde gracias a los diferentes contrastes presentados se desarrollan mayores oportunidades de solución innovadoras de problemas recurrentes en la sociedad que den cuenta a la reducción de desigualdad y la pobreza presente en el territorio.

\section{Referencias}

Agudelo, Z. M. L. (2016). Los Ecosistemas de Emprendimiento como parte de la Estrategia de Desarrollo de una Región. Universidad EAFIT, 122. http://hdl.handle.net/10784/11496

Alba, C. (2015). Business Incubator Model: An Approach. Revista Perspectivas, 65-90.

Alejandro Barrera Escobar, L. A. (2005). Estudio de Política Pública para el Emprendimiento y el Crecimiento Empresarial en Manizales. Manizales.

Almus, M. (2002). What characterizes a fast-growing firm? Jornal Applied Economics, 1497-1508.

Ayala García, J., \& Meisel Roca, A. (2016). La exclusión en los tiempos del auge : el caso de Cartagena. Documentos de Trabajo Sobre Economía Regional y Urbana.

Banco de la Republica. (Diciembre de 2016). www.banrep.gov.co. Obtenido de www.banrep.gov.co: http://www.banrep.gov.co/es/inversion-directa

Banco Mundial. (Junio de 2016). Obtenido de http://espanol.doingbusiness.org/rankings

Baumol, W. J. (1993). Entrepreneurship, Management, and the Structure of Payoffs. Cambridge: Cambridge: The MIT Press.

Becerra, G. P. (2010). Globalization and entrepreneurial growth through internationalization. ( La globalización y el crecimiento empresarial a través de estrategias de internacionalización). Revista cientifica Pensamiento y Gestión. ISSN: 1657-6276., 175 - 195.

Birch, D. L. (1987). Job Creation in America: How Our Smallest Companies Put the Most People to Work. New York: Free Press.

Business, I. D. (2017). Doing Business. Obtenido de espanol.doingbusiness.org

Cámara de Comercio de Cartagena. (Diciembre de 2017). Cartagena en cifras. Cartagena: Cámara de Comercio de Cartagena. https://www.banrep.gov.co/: https://www.banrep.gov.co/docum/Lectura_finanzas/pdf/dtser_178.pdf

Cámara de comercio de Cartagena. (2019). Cartagena en cifras. Cartagena: Centro de Estudios Económicos y Empresariales.

Cámara de comercio de Cartagena. (2020). Informe económico de los municipios de la jurisdiccion de la cámara de comercio de Cartagena. Cartagena: Centro de Estudios para el Desarrollo y la Competitividad-CEDEC.

Cepal. (2019). La Inversión Extranjera Directa en América Latina y el Caribe.

Departamento Administrativo Nacional de Estadisticas, DANE. (2015). Gran Encuesta Integrada de Hogares.

Félix Blásquez Santana, J. A. (2006). Factores explicativos del crecimiento empresarial. Especial referencia a las pequeñas y medianas empresas. Innovar. Revista de Ciencias Administrativas y Sociales, 43-56. 
GEM. (2012). Reporte Colombia Región Caribe 2012 - 2013. Cartagena: GEM Caribe.

GEM. (s.f.). Global Entrepreneurship Monitor . Global Entrepreneurship Monitor, https://gemcolombia.org/gem/.

Hidalgo, G., Kamiya, M., \& Reyes, M. (2014). Emprendimientos Dinámicos en América Latina. CAF.

Innpulsa. (2016). Ecosistemas regionales de emprendimiento en Colombia. Colombia.

Isenberg, D. (2010). Harvard Business Review. Obtenido de https://hbr.org/2010/06/the-big-ideahow-to-start-an-entrepreneurial-revolution

Isenberg, D. (2010). Proyecto BEEP-Babson Entrepreneurship Ecosystem Project. http://entrepreneurial-revolution.com/contact-us/.

Izquierdo, F. G. (2013). Características del emprendimiento con innovación en la ciudad de cartagena de indias.

Juan Carlos Díaz Casero, Ricardo Hernández Mogollón y David Urbano Pulido. (2005). Teoría económica institucional y creación de empresas. Vol. 11, $N^{\circ} 3$. ISSN: 1135-2533, $209-230$.

Kantis, H., Federico, J., \& Menendez, C. (2012). Políticas De Fomento Al Emprendimiento Dinámico En América Latina : Tendencias Y Desafíos. Caf, 1-50. http://www.caf.com/media/4233/politicas-emprendimiento-dinamico-america-latina.pdf

Kantis, H., Federico J, \& Garcia S.I. (2016). Condiciones sistémicas para el emprendimiento dinámico 2016: Novedades y tendencias para fortalecer e integrar los ecosistemas de la región.

Kelley, D, Bosma, N \& Amorós J. (2010). Global Report. Global Entrepreneurship Monitor 2010.

Llamas Fernández, F. J., \& Fernández Rodríguez, J. C. (2018). La metodología Lean Startup: desarrollo y aplicación para el emprendimiento. Revista EAN(84).

Ministerio de Comercio Industria y Turismo. (s.f.). http:/www.mincit.gov.co/minindustria/publicaciones/16435/politica_nacional_de_emprendimiento

Ministerio de Comercio, Industria y Turismo - MINCIT. (s.f.). MINCIT. Recuperado el Agosto de 2017, de http://www.mincit.gov.co/minindustria/publicaciones/16400/Definiciones

Moore, J. F. (1993). Predators and Prey: A New Ecology of Competition. Harvard Business Review, $71(3)$

North, D. (1990). Institutions, institutional change and economic performance. Cambridge: Cambridge University Press.

North, D. C. (1990). Institutions, institutional change and economic performance. Cambridge university press.

Orozco, T. J. A., \& Arraut, C. L. C. (2013). El entorno competitivo para el emprendimiento en la región Caribe de Colombia: caso de Barranquilla, Cartagena, Santa Marta y Sincelejo. Revista EAN, (74), 86-105. https://doi.org/10.21158/01208160.n74.2013.738

Ramos, C., Martínez, A., \& Fernández, R. (2004). Dianelt. Análisis de los factores determianntes de la creación de empresas: una evidencia empírica en Castilla y León. España: Proyecto de Investigación financiado por la orden EYE 1890/2004, de la Consejería de la Economia y Empleo de la Junta de Castilla y León.

Reinoso Lastra, J. F., \& Uribe, M. (2013). Emprendimiento y Empresarismo. Ediciones de la U. 
Romero, A. (Julio de 2017). Universidad del Externado. Obtenido de De Negocios: https://dernegocios.uexternado.edu.co/negociacion/la-importancia-del-emprendimiento-en-colombia/

Schumpeter, J. A. (1942). Capitalismo, Socialismo y Democracia. Madrid.

Schumpeter, J. A. (1949). Economic Theory and Entrepreneurial History. Reprinted from Change and the Entrepreneur. Cambridge: Cambridge: Harvard University Press. En Essays On Entrepreneurs, Innovations, Business Cycles, and the Evolution of Capitalism, Richard Clemence. New Brunswick: Transaction Publishers.

Sen, A. (1999). Development as Freedom. New York: OXFORD universiy press.

Steve Blank, Ben Horowitz, Eric Ries. (2015). $3 x 3$ Minute Summary Mashup of Business Startup with Steve Blank, Ben Horowitz and Eric Ries. Thimbles of Plenty Group.

Torres, C. V. (2016). A Teoria de Valores Refinada: associações com comportamento e evidências de validade discriminante e preditiva.

vamos, C. c. (2016). Cartagena cómo vamos. Obtenido de Cartagena cómo vamos: https://www.cartagenacomovamos.org/nuevo/aumento-la-pobreza-en-cartagena-en-2016/

Vásquez, M. L. (2013). Políticas Públicas de Emprendimiento en Colombia. Cali: Universidad autónoma de occidente, facultad de ciencias económicas administrativas, departamento de ciencias económicas..

Vesga, R. (2009). Emprendimiento E Innovación en Colombia: ¿Que nos esta haciendo falta? Bogotá D.C.

Villanueva Imitola, A. (2017). Competitividad empresarial de los pequeños y medianos hoteles ubicados en la ciudad de Barranquilla. Saber, Ciencia Y Libertad, 12(2), 99-106. https://doi.org/10.18041/2382-3240/saber.2017v12n2.1576

Zambrano-Vargas, S. M., \& Vázquez-García, A. W. (2019). Algunas perspectivas teóricas para el estudio del emprendimiento y el género. Saber, Ciencia Y Libertad, 14(1), 159-170. https://doi.org/10.18041/2382-3240/saber.2019v14n1.5216

Zoltan Acs and Catherine Armington. (2006). Entrepreneurship, Geography, and American Economic. Inglaterra: Cambridge University Press.

Zoltan Acs, C. A. (2006). Entrepreneurship, Geography, and American Economic. Inglaterra: Cambridge University Press. 